\title{
ON THE PROBLEM OF BROWNIAN MOTION OF NON-LINEAR SYSTEMS
}

\author{
by C. T. J. ALKEMADE \\ Fysisch Laboratorium van de Rijks-Universiteit te Utrecht, Nederland.
}

\section{Summary}

A kinetic derivation is given of the spectral noise intensity in first order approximation and for relatively high frequencies only, for a non-linear RC-circuit consisting of an idealized diode-valve in thermal equilibrium and a condenser. The discrepancy with the results obtained by other authors (MacDonald, 1957 and Van Kampen, 1958) on the basis of a very general hypothesis, in which the mechanism of the noise-generating element is not kinetically specified, is discussed.

1. Introduction. The discussion of the brownian motion of non-linear systems has recently been revived by the publications of MacDonald ${ }^{1}$ ) and Van Kampen ${ }^{2}$ ). Both authors derived an expression for the electrical fluctuations in a RC-circuit in thermal equilibrium with a temperature bath, in which $R$ was assumed to depend slightly on the voltage across the condenser. In their derivations the kinetics of the noise-generating element was not considered, but use was made of general hypotheses in which the observable circuit characteristics served as the specifying element. The discrepancy between the results of MacDonald and Van Kampen was discussed by the latter ${ }^{2}$ ).

As was pointed out by the authors, a justification of their hypotheses by noise experiments with non-linear elements seems to be hardly feasible, for the time being. We have now tried to check their results by comparing them with the theoretical result obtained in a kinetic derivation for a case of modest non-linearity, in which the mechanism of the noise-generating element could easily be specified.

2. Analysis. We consider the specific case of a vacuum diode-valve consisting of two plane, parallel electrodes of large surface area, opposite to each other at a short distance.

Both electrodes are assumed to be in equilibrium with a temperature bath. The work functions of the two electrodes are assumed to be different and are chosen in such a way that noticeable thermal emission of electrons 
occurs from both plates at the prevailing temperature. The diode is connected to a condenser with capacity $C$ (the capacity of the diode itself may be comprised in $C$ ). We idealize the diode by assuming that the electrons in the diode vacuum space do not interfere with each other (no noticeable space charge) and that the transit time of the electrons is practically zero. So we exclude the region of high frequencies at which transit time effects influence the noise spectrum.

The $i-v$ characteristic of this diode (which may be considered as a rectifier) may be given by:

$$
i=I_{0}(\exp [e v / k T]-1)
$$

in which $i$ is the net current through the valve, $v$ is the applied voltage, $k$ is the Boltzmann constant, $T$ is the absolute temperature, $e$ is the absolute value of the electron charge, and $I_{0}$ is the (average) current of electrons emitted by the plate with the higher work-function. $I_{0}$ is assumed to be independent of $v$ as long as $v$ is sufficiently small.

In second order approximation (the voltage-fluctuations are assumed to be small!) we may write:

$$
i=I_{0}\left\{e v / k T+\frac{1}{2}(e / k T)^{2} v^{2}+\frac{1}{6}(e / k T)^{3} v^{3}\right\} .
$$

Evidently we here have a case of (modest) non-linearity with, in second order approximation:

$$
G(v) \equiv i / v=G(0)\left\{1+\frac{1}{2}(e / k T) v+\frac{1}{6}(e / k T)^{2} v^{2}\right\}
$$

. with $G(0) \equiv e I_{0} / k T$. In the case of the diode shunted by a condenser, we have: $v=q / C$, if $q$ is the charge on the condenser.

Owing to the assumed absence of mutual interference, the noise in the diode current can be calculated by square summation of the noise contributions from the opposing electron flows. For the electrons emitted by the plate with the higher work function the diode operates under saturation conditions, so the spectral intensity of the noise contributed by these electrons is given by:

$$
S_{1}=2 e I_{0}
$$

For the electrons emitted by the opposite plate the diode operates in the exponential region. So the chance that an electron emitted arrives at the other plate is certainly less than unity and depends on the voltage across the diode, i.e., on the charge of the condenser. Because the system is in thermal equilibrium, the charge on the condenser is essentially subject to fluctuations with:

$$
\overline{q^{2}}=k T C .
$$

MacDonald ${ }^{1}$ ) has argued the validity of this expression for the case in which the dissipative element is non-linear. 
The fluctuation of the condenser charge modulates, as it were, the chance for the electrons emitted by the electrode with the lower work function to escape to the other electrode. The non-linearity of the dissipative element (here the diode valve) is essentially accounted for in the noise calculation by allowing for this modulation effect. In the derivation to be given below the basic assumption is made that during any short time interval in which the condenser charge (voltage) does not change noticeably, the statistics of the electrons arriving at the electrode with the higher work function, is uniquely determined by the then prevailing voltage across the diode. The fact that in the case in question this voltage is determined by the (fluctuating) charge on the condenser, and not, for instance, by a battery, is believed not to be relevant at all.

The spectral noise intensity $S_{2}$ of the current of electrons arriving at the electrode with the higher work function, may be derived by considering a long time interval $T_{0}$ and by expanding the current pulses caused by the transition of the separate electrons into a Fourier series. The component of the total fluctuating current with frequency $f=n / T_{0}$ ( $n$ is an integer) is then found to be:

$$
\begin{aligned}
i_{f}(t)=\sum_{p=1}^{N} A_{n}\left\{\exp \left[-\mathrm{j} 2 \pi n t_{p} / T_{0}\right] \cdot \exp \left[\mathrm{j} 2 \pi n t / T_{0}\right]\right\}+ \\
+A_{-n}\left\{\exp \left[\mathrm{j} 2 \pi n t_{p} / T_{0}\right] \cdot \exp \left[-\mathrm{j} 2 \pi n t / T_{0}\right]\right\},
\end{aligned}
$$

with $A_{-n}=A_{n}{ }^{*}$ and $A_{n} \cdot A_{n}{ }^{*}=e^{2} / T_{0}{ }^{2}$, if during time interval $T_{0}, N$ electrons have arrived at the opposite plate at the moments $t_{1}, t_{2}, t_{3} \ldots$ $t_{p} \ldots t_{N}$.

$S_{2}$ is then found from: $S_{2}=\overline{\overline{i_{f}(t)^{2}}} \cdot T_{0}$. In this expression the first bar denotes the average over the interval $T_{0}$, and the second bar denotes the average over an ensemble of diodes under identical macroscopic conditions, taken in the same interval $T_{\mathbf{0}}$. One has:

$$
\overline{\overline{i_{f}(t)^{2}}}=\left(\int_{0}^{T_{0}}{\overline{i_{f}(t)^{2}}}^{\text {ens. }} \mathrm{d}\right) / T_{0}=2 A_{n} A_{n} * \cdot{\overline{R \cdot R^{*}}}^{\text {ens. }}=\left(2 e^{2} / T_{0}{ }^{2}\right) \cdot{\overline{R R^{*}}}^{\text {ens. }}
$$

with

$$
R \equiv \Sigma_{p=1}^{N} \exp \left[\mathrm{j} 2 \pi n t_{p} / T_{0}\right]
$$

Each term in $R$ may be represented by a point on a circle with unit radius in the complex plane, having an argument equal to $2 \pi n t_{p} / T_{0}$ modulo $2 \pi$.

We now restrict ourselves to frequency values $f$ so high that during any time interval $(t ; t+1 / f)$ the charge of the condenser is practically constant (say: $f \gg G(0) / C$ ). Of course, $f$ should not be so high that transit time effects would be noticeable. This condition can always be met by choosing suitable dimensions and conditions for the diode-valve.

We now introduce the probability function $P(q)$ in such a way that $P(q) . \Delta t$ denotes the probability that during an infinitesimal short time interval $\Delta t$ an electron transition takes place to the electrode with the higher 
work function. This probability is a function of the condenser charge $q$, as the average fraction of emitted electrons arriving at the opposite electrode depends on the voltage across the diode. The average number $P(q)$ of electrons arriving per sec at the electrode considered, with given value of $q$, is given by:

as: $v=q / C$.

$$
P(q)=I_{0} \exp [e q / k T C] / e
$$

Consider a time interval $m / f ;(m+1) / f$, with $f$ so high that the above mentioned condition is met. Then $P(q)$ will be practically constant during this interval, and may be considered equal to $P\left(q_{m}\right)$ if $q_{m}$ is the average value of $q$ during the interval. As the thermal emission of electrons is completely at random, the distribution in time of the electron transitions during this interval will obey Poisson's law. Likewise the points in the complex plane, representing terms $\exp \left[\mathrm{j} 2 \pi n t_{p} / T_{0}\right]$, with $m / f<t_{p}<(m+1)$ if will be distributed along the circumference of the unit circle according to Poisson's law, with an average density $P\left(q_{m}\right) / 2 \pi f$ (per unit angle). Since this holds for any time-interval $m / f ;(m+1) / f$, with $m=1,2, \ldots n$, the distribution of points from the whole interval $\left(0 ; T_{0}\right)$ along the unit circle will also obey the Poisson law, with an average density equal to

$$
\sum_{m=1}^{n} P\left(q_{m}\right) / 2 \pi f
$$

It is now easily proved that:

$$
{\overline{R R^{*}}}^{\text {ens. }}=2 \pi{\overline{\left(\sum_{m=1}^{n} P\left(q_{m}\right) / 2 \pi f\right)}}_{\text {ens. }}^{\text {. }}
$$

so that from (3):

$$
S_{2}=2 e^{2}{\overline{\sum_{m=1}^{n} P\left(q_{m}\right) / n}}^{\mathrm{ens}}
$$

as:

$$
f . T_{0}=n .
$$

Evidently, the net transport of charge through the diode-valve during time interval $T_{0}$, when averaged over an ensemble of identical systems, should equal zero. Since all electrons emitted by the electrode with the higher work function arrive at the opposite electrode, the average current of these electrons must equal the average emission rate $I_{0}$ of that electrode. We thus also have for the electrons flowing in the opposite direction:

so that:

$$
e \overline{\sum_{m=1}^{n} P\left(q_{m}\right) / n}=I_{0}
$$

$$
S_{2}=2 e I_{0}
$$

The spectral noise intensity in the total diode current is now given by:

$$
S=S_{1}+S_{2}=4 e I_{0}=4 G(0) k T .
$$

It should be remembered that $(6)$ is the limit of the spectral noise intensity 
for $f \rightarrow \infty$. This result is independent of the approximation made in (1). Making use of (4) and (5) we also find:

$$
\overline{\exp [e q / k T C]}=1
$$

or, in first order approximation:

or:

$$
(e / k T C) \bar{q}+\frac{1}{2}(e / k T C)^{2} \overline{q^{2}}=0
$$

$$
\bar{q}=-\frac{1}{2} e,
$$

because $\overline{q^{2}}=k T C$ (cf. above)

It then follows from (1) and (7) that:

$$
\vec{G} \simeq G(0)\left(1-e^{2} / 12 k T C\right)
$$

Combining (6) and (8):

$$
S=4 G(0) k T \simeq 4 \bar{G} k T\left(1-e^{2} / 12 k T C\right)^{-1}
$$

Expressing $S$ in terms of the average conductance of the diode-valve, the usual Nyquist formula is found provided with a correction factor $\left(1-e^{2} / 12 k T C\right)^{-1}$ due to the non-linearity effect. This result is only approximately valid if $e^{2} / 12 k T C \ll 1$, which may be expected to hold true under all practical conditions.

3. Discussion. MacDonald ${ }^{1}$ ) has applied his noise formula for modest non-linearity to the case given by our equation (1), which describes our exponential diode in second order approximation. As a result the spectral noise intensity of the diode current is found to behave as:

$$
S \rightarrow 4 G(0) k T\left(1-3 e^{2} / 4 k T C\right) \text { for } t \rightarrow \infty \text {. }
$$

When applying the noise formula for modest non-linearity given by Van Kampen ${ }^{2}$ ) to the case defined by our equation (1), it is found that:

$$
S \rightarrow 4 G(0) k T\left(1+e^{2} / 6 k T C\right) \text { for } f \rightarrow \infty .
$$

In both these formulas terms of higher order are neglected, as $e^{2} \ll k T C$. Both formulas (10) and (11) differ from each other and from our formula (6). It is not believed that an error is introduced in (6) by the approximation, made in the derivation, that the condenser charge does not vary during a time interval $1 / f$ if $t$ is sufficiently high. A more elaborate derivation for a very similar case (3), in which the condenser charge is allowed to vary to a limited extent during an interval $1 / f$, confirms the result of the more elementary derivation, as given above, for $f \rightarrow \infty$.

The discrepancy of our formula with formula (10) may be related to the discussion given by Van Kampen ${ }^{2}$ ) on the basic assumptions of Litt. ${ }^{1}$ ).

The reason of the discrepancy with formula (11) does not seem so easily: Physica XXIV 
traced. It may be noted that according to $\mathrm{Van} \mathrm{Kampen}{ }^{2}$ ) the distribution law of the fluctuating charge $q$ on the condenser in equilibrium should be the normal law:

$$
G(q)=(2 \pi k T C)^{-t} \cdot \exp \left[-q^{2} / 2 k T C\right],
$$

also when the dissipative element is non-linear. MacDonald ${ }^{1}$ ) found, as we did (cf. (7)), that $\bar{q}=-\frac{1}{2} e$, however. This point of difference might be significant for an explanation of the discrepancy under discussion.

Care should be taken in handling the distribution function for the condenser charge. Since we have assumed in our derivation that the duration of an electron transition is infinitely small, the distribution function of the condenser charge should be essentially discontinuous in our case. For this reason application of the normal distribution law is strictly not allowed.

Both MacDonald and Van Kampen derived their formulas in a nonmolecular way, without considering the "kinetics" of the brownian motion. The dissipative element is specified in their papers by some characteristic function of the condenser charge $(G(q)$ and $\xi(q)$ respectively, where $G(q)$ is operationally defined, and $\xi(q)$ is derived from the Fokker-Planck equation). An essential step in their derivations is the interpretation of these functions in terms of the observable conductance of the non-linear element under consideration. It seems to me that this interpretation is not always unambiguous and in fact again includes a hypothesis. With regard to the discrepancies noted above, it might be interesting to clarify this point.

Received 14-8-58.

\section{REFERENCES}

1) MacDonald, D. K. C., Phys. Rev, 108 (1957) 541.

2) Van Kampen, N. G., Phys. Rev. 110 (1958) 319.

3) Alkemade, C. T. J., Internal report, Utrecht, i 140. 